\title{
Selbstständigkeit im Alltag
}

\section{Acht Module für ein neues Begutachtungsverfahren zur Einschätzung von Pflegebedürftigkeit}

\author{
Klaus Wingenfeld, Andreas Büscher und Barbara Gansweid
}

Dr. Klaus Wingenfeld ist wissenschaftlicher Geschäftsführer, Dr. Andreas Büscher ist Mitarbeiter des Instituts für Pflegewissenschaft an der Universität Bielefeld (IPW).

Dr. Barbara Gansweid arbeitet beim Medizinischen Dienst der Krankenversicherung (MDK) Westfalen-Lippe. E-Mail andreas.buescher@uni-bielefeld.de klaus.wingenfeld@uni-bielefeld.de bgansweid@mdk-wl.de
Die bisherige Methode zur Einschätzung der Pflegebedürftigkeit steht seit langem in der Kritik. Ein neu entwickeltes Verfahren stellt nun nicht mehr die aufgewendete Pflegezeit in den Mittelpunkt der Entscheidung, sondern die Selbstständigkeit eines Menschen bei seinen Alltagsaktivitäten und bei der Gestaltung seiner Lebensbereiche.

Seit Herbst des Jahres 2006 sind verstärkte Bemühungen zu verzeichnen, eine sozialrechtliche Neufassung des Begriffs der Pflegebedürftigkeit vorzubereiten und parallel dazu ein neues Begutachtungsverfahren zu erarbeiten (vgl. den Beitrag »Gesundheitlich bedingte Beeinträchtigung der Selbstständigkeit und auf personelle Hilfe angewiesen « in diesem Heft).

Im Frühjahr 2007 erfolgte die Ausschreibung eines entsprechenden Entwicklungsprojekts nach $\mathbb{S} 8$ Abs. 3 SGB XI durch die Spitzenverbände der Pflegekassen. Auf diese Ausschreibung hat sich eine Arbeitsgemeinschaft aus Wissenschaftlern und Vertretern des Medizinischen Dienstes der Krankenversicherung (MDK) erfolgreich beworben. Anfang März 2008 wurde der Prototyp eines neuen Begutachtungsverfahrens vorgelegt, das im Rahmen des Projektes gemeinsam vom Institut für Pflegewissenschaft an der Universität Bielefeld (IPW) und vom Medizinischen Dienst der Krankenversicherung Westfalen-Lippe (MDK WL) erarbeitet worden ist. Mitglieder der Arbeitsgemeinschaft sind außerdem der Medizinische Dienst der Spitzenverbände der Krankenkassen e. V. und das Institut für Public Health und Pflegeforschung der Universität Bremen, die das neue Instrument in einer zweiten, bis Oktober 2008 laufenden Projektphase einer umfassenden Erprobung unter den praktischen Bedingungen der Begutachtung unterziehen.

Gegenüber dem bisherigen Begutachtungsverfahren gibt es viele Veränderungen, von denen zwei besonders wichtig sind, weil sie eine grundlegende Umorientierung im Verständnis von Pflegebedürftigkeit beinhalten:

- Das neue Instrument zielt auf eine umfassende Berücksichtigung von Pflegebedürftigkeit, vermeidet also die Reduzierung von Pflegebedürftigkeit auf Hilfebedarf bei bestimmten Alltagsverrichtungen, die für die heutige soziale Pflegeversicherung (SGB XI) charakteristisch ist. Es erfasst sowohl körperliche Beeinträchtigungen als auch kognitive und psychische Einbußen und Verhaltensauffälligkeiten, die einen besonderen Unterstützungsbedarf nach sich ziehen.

- Im Unterschied zum jetzigen Begutachtungsverfahren ist der Maßstab zur Einschätzung von Pflegebedürftigkeit nicht die erforderliche Pflegezeit, sondern der Grad der Selbstständigkeit bei der Durchführung von Aktivitäten oder der Gestaltung von Lebensbereichen.

Derjenige Teil des neuen Begutachtungsassessments, mit dem der Grad der individuellen Beeinträchtigungen ermittelt wird, ist in insgesamt acht Module gegliedert. Jedes Modul umfasst eine Gruppe artverwandter Aktivitäten, Fähigkeiten oder einen Lebensbereich und enthält mehrere Unterpunkte (»Items « oder »Merkmale«), zu denen der Gutachter eine Einschätzung liefern soll:

1. Mobilität: Fortbewegung über kurze Strecken und Lageveränderungen des Körpers.

2.Kognitive und kommunikative Fähigkeiten: Gedächtnis, Wahrnehmung, Denken, Kommunikation (geistige und verbale »Aktivitäten $«)$.

3. Verhaltensweisen und psychische Problemlagen: Verhaltensweisen, die mit einer Selbstgefährdung oder mit der Gefährdung anderer verbunden sein oder andere Probleme mit sich bringen können, sowie psychische Probleme wie Ängstlichkeit, Panikattacken oder Wahnvorstellungen (Selbstständigkeit 
im Umgang mit inneren Handlungsimpulsen und Emotionen).

4.Selbstversorgung: Körperpflege, sich Kleiden, Essen und Trinken sowie Verrichtungen im Zusammenhang mit Ausscheidungen.

5.Umgang mit krankheits- und therapiebedingten Anforderungen und Belastungen: Aktivitäten, die auf die Bewältigung von Anforderungen und Belastungen infolge von Krankheit oder Therapiemaßnahmen zielen, z. B. Medikamenteneinnahme, Wundversorgung, Umgang mit körpernahen Hilfsmitteln oder Durchführung zeitaufwendiger Therapien innerhalb und außerhalb der häuslichen Umgebung.

6. Gestaltung des Alltagslebens und soziale Kontakte: Einteilung von Zeit, Einhaltung eines Rhythmus von Wachen und Schlafen, sinnvolles (bedürfnisgerechtes) Ausfüllen von verfügbarer Zeit und Pflege sozialer Beziehungen.

7.Außerhäusliche Aktivitäten: Teilnahme an sozialen und im weitesten Sinne kulturellen Aktivitäten (einschließlich außerhäusliche Mobilität).

8.Haushaltsführung: hauswirtschaftliche Tätigkeiten und Regelung der für die alltägliche Lebensführung notwendigen geschäftlichen Belange (Nutzung von Dienstleistungen, Umgang mit Behörden, Geldangelegenheiten etc.).

Die Anwendung des neuen Verfahrens liefert für jedes Modul ein Einschätzungsergebnis, das für sich genommen Aussagekraft besitzt (z. B. Modul 1: Grad der Selbstständigkeit bei Aktivitäten, die der Fortbewegung oder Lageveränderung des Körpers dienen). Erst in einem zweiten Schritt werden die Teilergebnisse nach bestimmten, vorgegebenen Berechnungsregeln (Bewertungssystematik) zu einem Gesamtergebnis zusammengeführt. Zur Ermittlung einer Stufe der Pflegebedürftigkeit geschieht dies für die Ergebnisse der Module 1 bis 6, die zusammen als Wert auf einer Skala zwischen 0 und 100 Punkten dargestellt werden. Diese Skala wiederum ist in mehrere Bereiche unterteilt, die jeweils einer bestimmten Stufe der Pflegebedürftigkeit zugeordnet sind. Die Module 7 und 8 werden gesondert berücksichtigt und dienen dazu, in ähnlicher Weise einen Grad der Hilfebedürftigkeit zu ermitteln. Das Verfahren unterscheidet also zwischen Hilfe- und Pflegebedürftigkeit.
Im Falle der Pflegebedürftigkeit sieht das Verfahren eine Unterteilung in fünf Stufen vor. Sie ist so angelegt, dass auch solchen Pflegebedürftigen eine Stufe zugeordnet wird, die relativ geringe Beeinträchtigungen aufweisen und nach den heute geltenden Regelungen der Pflegeversicherung keine Leistungen erhalten. Damit soll vermieden werden, dass Menschen als » nicht pflegebedürftig « bezeichnet werden, obwohl sie auf pflegerische siken). Zum anderen soll der Gutachter eine Einschätzung zum Erfordernis rehabilitativer Maßnahmen vornehmen und eine entsprechende Empfehlung abgeben.

Beides ist zwar auch heute schon Bestandteil des Begutachtungsverfahrens, allerdings sieht das neue Instrument eine wesentlich systematischere Erfassung und Einschätzung vor. So soll der Gutachter in den Modulen 1. »Mobilität «, 2. »Kog-

\section{»Sinnvoll ist eine Unterscheidung zwischen Hilfebedürftigkeit und Pflegebedürftigkeit «}

Hilfe angewiesen sind. Inwieweit allerdings mit geringen Beeinträchtigungen ein Leistungsanspruch gegeben sein soll, wird am Ende sozialpolitisch zu entscheiden sein.

Die Einschätzung der Pflegebedürftigkeit von Kindern erfolgt mit dem gleichen Erfassungsbogen. Die wesentlichen Unterschiede zum Verfahren bei Erwachsenen betreffen die Bewertungssystematik, also die Methode, nach der Pflegebedürftigkeit bestimmt wird. Das Ergebnis der Einschätzung beschreibt in diesem Fall nicht den Grad der Selbstständigkeit, sondern dessen Abweichung von der Selbstständigkeit gesunder, altersentsprechend entwickelter Kinder. Außerdem bleibt das Modul 8 "Haushaltsführung « außer Betracht. Die Festlegung der Altersgrenzen, die dabei verwendet werden, stützt sich auf eine breit angelegte Recherche und Auswertung von Studien zur kindlichen Entwicklung sowie auf Unterscheidungen alterstypischer Entwicklungsstufen, die sich in anderen Einschätzungsinstrumenten finden.

\section{Systematische Einschätzung von Fragen zur Prävention und Rehabilitation}

Im Anschluss an die Module gibt es zwei weitere wichtige Abschnitte. Dazu gehört zum einen die explizite Erfassung wesentlicher, präventionsrelevanter Risiken (krankheitsbedingte Risiken, Umweltfaktoren und verhaltensbedingte $\mathrm{Ri}$ - nitive und kommunikative Fähigkeiten «, 4. »Selbstversorgung « und 5. »Umgang mit krankheits- und therapiebedingten Anforderungen « die Entwicklungstendenz der Selbstständigkeit und Fähigkeiten einschätzen. Er beantwortet hierbei auch die Frage, ob ein höherer Grad an Selbstständigkeit wieder erlangt werden kann oder ob es Möglichkeiten gibt, einer Verschlechterung entgegenzuwirken. Der Gutachter soll außerdem angeben, welche Möglichkeiten der Verbesserung existieren, beispielsweise durch die Optimierung therapeutischer Maßnahmen, die Optimierung der räumlichen Umgebung, durch Hilfsmitteleinsatz und dessen Optimierung oder durch andere Maßnahmen. Auf dieser Grundlage und unter Berücksichtigung etwaiger Einschränkungen der Rehabilitationsfähigkeit soll eine explizite Empfehlung zur Einleitung von Rehabilitationsmaßnahmen formuliert werden. Bei negativer Entscheidung sind vom Gutachter entsprechende Begründungen gefordert.

Noch während der Entwicklungsphase wurde mit einer Entwurfsversion des neuen Instruments ein Pretest durchgeführt (100 Erwachsene und 41 Kinder). Hierbei zeigte das neue Einschätzungsverfahren eine überzeugende Praktikabilität. Die Methodik, mit der das neue Instrument operiert, führt zu plausiblen Ergebnissen. Die Antragsteller und ihre Angehörigen signalisierten Aufgeschlossenheit gegenüber dem neuen Verfahren, da die mit ihm erfassten Sachverhalte ihrem Alltagserleben stärker gerecht werden als das heutige Begutachtungsverfahren. 
Auch das Ziel, kognitive Beeinträchtigungen und psychische Störungen adäquat zu berücksichtigen, kann den Ergebnissen des Pretests zufolge erreicht werden. Personen, die keine oder nur geringfügige körperliche, aber deutlich ausgeprägte kognitive Probleme aufweisen und daher auf eine intensive Begleitung im Lebensalltag angewiesen sind, gelten innerhalb der neuen Stufensystematik größtenteils als erheblich beeinträchtigt. Dadurch dass kognitive oder psychische Beeinträchtigungen als eigenständiger Faktor gewichtet werden, ergibt sich umgekehrt, dass die Selbstständigkeitseinbußen von Personen mit ausschließlich körperlichen Beeinträchtigungen weit fortgeschritten sein müssen, um hohe Punktwerte und damit eine hohe Stufe zu erreichen. Das neue Verfahren ist allerdings so konstruiert, dass sich daraus keine neuen Schieflagen oder Benachteiligungen einzelner Personengruppen ergeben.

\section{Resümee}

Mit dem neuen Instrument liegt ein vielversprechender und praktikabler neuer Ansatz zur Feststellung von Pflegebedürftigkeit vor. Das Verfahren befindet sich derzeit in einer umfangreichen praktischen Erprobung, die in mehreren Bundesländern von den dortigen Medizinischen Diensten durchgeführt wird. Sollte diese Erprobung zu positiven Ergebnissen führen und gelänge darüber hinaus eine Harmonisierung zwischen dem neuen Verfahren und der Neufassung des sozialrechtlichen Begriffs der Pflegebedürftigkeit, so bestünden gute Aussichten auf eine Behebung von zentralen, seit vielen Jahren intensiv diskutierten Problemen der Pflegeversicherung.

\section{Literatur}

Wingenfeld, K./Büscher, A./Gansweid, B. (2008): Das neue Begutachtungsssessment zur Feststellung von Pflegebedürftigkeit. Abschlussbericht und Anlagenband zur Hauptphase 1: Entwicklung eines neuen Begutachtungsinstruments. Bielefeld. Im Internet verfügbar unter http://www.vdak.de/vertragspartner/Pflegeversicherung/Modell programm/Projekte/modellprg_projekt_16/index.htm

\section{"Einer der besten seiner Zunft.»}

Prof. Dr. H.-D. Gottlieb, Sozialrecht aktuell 1/99, zur Vorauflage

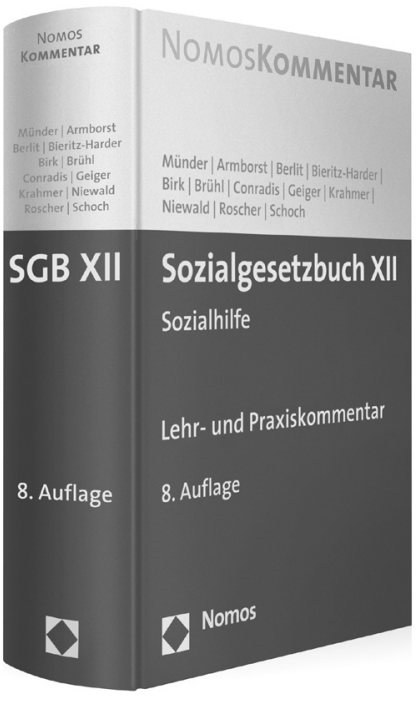

\section{Sozialgesetzbuch XII \\ Sozialhilfe}

Lehr- und Praxiskommentar

Von Prof. Dr. Johannes Münder, MinR Christian Armborst, RiBVerwG Prof. Dr. Uwe Berlit, Prof. Dr. Renate Bieritz-Harder, Prof. Dr. Ulrich-Arthur Birk, Prof. Dr. Albrecht Brühl, RA Dr. Wolfgang Conradis, FASozR, RiSG Udo Geiger, Prof. Dr. Utz Krahmer, RiLSG Stephan Niewald, Prof. Dr. Falk Roscher und RegDir a.D. Dietrich Schoch

8. Auflage 2008, 1.017 S., geb., 44,- $€$, ISBN 978-3-8329-2930-5

Die Neuauflage des LPK-SGB XII bringt Sie im Sozialhilferecht wieder auf den neuesten Stand.

"Auf diesen angesichts seines Umfangs und seiner Qualität verhältnismäßig sehr preiswerten Lehr- und Praxiskommentars sollte keine Sozialberaterin und kein Sozialberater verzichten; denn es zeichnet sich dadurch aus, dass es von hervorragenden Sachkennern verfasst ist, die auch bei unklarer Rechtslage stets Wege aufzeigen, wie Sozialhilfe ein menschenwürdiges Leben sichern muss."

Recht-Informationsdienst der Zeitschrift Caritas, NRW 2/o8

"Eine unentbehrliche Arbeitshilfe. Wohl kein anderer Kommentar kann einen vergleichbaren gebündelten Sachverstand an Autoren aus Universität, Gerichten und der Anwaltschaft aufbieten«.

Rechtsdienst der Lebenshilfe 3/05, zur Vorauflage 